\title{
聚苯胺@碳纳米纤维复合材料对放射性核素铀的 高效去除
}

\author{
王苏菲 ${ }^{1}$, 于淑君 ${ }^{2}$, 吴忆涵 ${ }^{2}$, 庞宏伟 $^{2}$, 陈中山 ${ }^{2}$, 王祥学 $3^{*}$ \\ 1. 北京师范大学附属实验中学, 北京 100032 \\ 2. 华北电力大学环境科学与工程学院, 北京 102206 \\ 3. 华北电力大学环境科学与工程系, 保定 071003 \\ *通讯作者, E-mail: xxwang@ncepu.edu.cn \\ 收稿日期：2018-05-30; 接受日期：2018-07-25; 网络版发表日期: 2018-10-31 \\ 国家自然科学基金(编号: 21607042)和中央高校基本科研业务费专项资金(编号: 2018ZD11, 2018MS114)资助项目
}

摘要本文通过原位聚合方法成功制备了聚苯胺改性的碳纳米纤维(PANI@CNF)复合材料, 并用于水溶液中 放射性核素铀(U(VI)) 的高效去除。扫描电子显微镜( SEM)、透射电子显微镜(TEM)、X射线衍射(XRD)、傅里叶 红外光谱(FTIR)和X射线光电子能谱(XPS)等表征证明所制备的材料具有丰富的官能团和优良的物理化学性质. 批实验方法系统研究了周围环境( $\mathrm{pH}$ 、背景电解液、反应时间和温度)的变化对U(VI)去除结果的影响. 结果表明, $\mathrm{pH}$ 对于 $\mathrm{U}(\mathrm{VI})$ 去除影响很大, 而离子强度没有影响, 表明二者之间的作用机理为内层表面络合. 吸附能够在 $30 \mathrm{~min}$ 内快速达到平衡, 且符合拟二级动力学模型. 吸附等温线符合Langmuir等温线, 表明U(VI)的去除是单分子层均匀 吸附过程。在 $\mathrm{pH}=5.0$ 和 $T=298 \mathrm{~K}$ 时, PANI@CNF对U(VI) 的最大吸附量高达 $319.4 \mathrm{mg} / \mathrm{g}$, 远远高于单纯的CNF $(133.9 \mathrm{mg} / \mathrm{g}) . \mathrm{U}(\mathrm{VI})$ 主要与材料表面的含氮和含氧官能团形成了稳定的内层络合物, 从而达到高效去除的目的. 以上分析表明, PANI@CNF具有快速反应动力学和高效吸附能力, 可以作为放射性核素高效去除的潜在储备材 料, 为我国核废料治理工作提供理论依据.

关键词碳材料, 聚苯胺, 铀, 光谱分析, 吸附

\section{1 引言}

随着人口的增长和化石能源的消耗，不断增加的 能源危机一直是主要问题和研究热点之一. 核能作为 一种清洁高效的裂变或聚变能源, 已经成为解决能源 危机的主要候选能源 ${ }^{[1,2]}$. 而铀资源则被认为是核能发 展的核心. 土壤、矿产和海水中含有大量的铀. 在过去
的几十年里, 铀矿开采和加工、核安全事故和不当的 核废料管理导致大量可溶U(VI)离子通过地表或地下 水系统的迁移进入自然环境 ${ }^{[3,4]}$. 由于长半衰期和高毒 性, U(VI)离子会导致细胞生物活性降低, 甚至导致癌

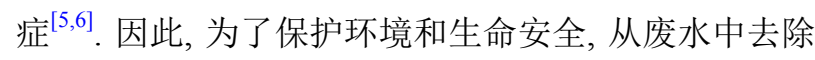
$\mathrm{U}(\mathrm{VI})$ 显得非常重要和紧迫.

许多先进的物理和化学方法, 如化学沉淀、固相

引用格式: Wang S, Yu S, Wu Y, Pang H, Chen Z, Wang X. Highly efficient removal of radioactive uranium on polyaniline modified carbon nanofiber composites. Sci Sin Chim, 2019, 49: 71-79, doi: 10.1360/N032018-00125 
萃取、离子交换、催化和膜分离等，已被用于从自然 环境中恢复和聚集放射性核素 ${ }^{[79]}$. 在这些方法中, 吸 附技术由于简单、经济有效、反应条件温和和环境友 好性, 被认为是去除放射性核素最经济、最方便的方 法 $^{[10]}$. 各种吸附剂，包括黏土矿物材料、碳基材料、 金属有机框架材料、零价铁材料和生物材料等，已被 广泛应用于废水中 $U(V I)$ 的去除 ${ }^{[1115]}$. 这些材料在放 射性核素的去除中表现出不同的物理化学性质和优缺 点. 例如，碳基材料具有丰富的含氧功能基团(如 $-\mathrm{COOH} ，-\mathrm{OH}$ 和 $-\mathrm{O}-$ )能够高效地去除放射性核素，但 是成本相对较高、合成过程也很复杂. 自然界中广泛 存在的黏土矿物材料具有较高的化学稳定性，但是去 除速率和吸附效率相对较低. 金属有机框架材料、零 价铁材料和生物材料也表现出优异的吸附效率和清除 能力，但是吸附性能极易受周围环境条件的影响。因 此，急需开发新型具有较好环境稳定性、较快去除速 率和较高吸附能力的材料用于放射性核素的去除.

近年来，由于性能优异，碳纳米纤维(CNF)已广泛 应用于各个领域。中国科学技术大学俞书宏课题 组 ${ }^{[16,17]}$ 开发了一种利用超长碲纳米线为模板、以葡萄 糖为碳源制备 $\mathrm{CNF}$ 的水热碳化技术，该方法的独特优 点是可以控制 $\mathrm{CNF}$ 的微观组织和物理化学性质. 由于 其优异的机械稳定性、较大的表面积和良好的亲水性 等特性，CNF被广泛应用于去除石油、有机污染物和 重金属离子等各种污染物 ${ }^{[18-20]}$. 本课题组 ${ }^{[21 ~ 23]}$ 在 CNF 对放射性核素和重金属离子的去除方向也进行了大量 的研究，发现CNF表面丰富的含氧官能团可以有效地 清除污染物. 但是, CNF也不可逆转地形成了聚集物, 大大缩小了其表面积并降低了去除能力. 目前，在水 溶液中减少 CNF聚集的有效方法仍然较少.

聚苯胺(PANI)由于含有大量的胺和亚胺功能团, 对重金属离子和放射性核素具有很强的亲和力 ${ }^{[24]}$ ，然 而，机械溶解度差和加工能力不强极大限制了它的单 纯实验研究和商业应用. 具有优良吸附性能的PANI复 合材料被大量研究并用于去除重金属离子, 如 $\mathrm{Hg}(\mathrm{II})$ 、 $\mathrm{Cd}(\mathrm{II}) 、 \mathrm{Cr}(\mathrm{VI}) 、 \mathrm{~Pb}(\mathrm{II})$ 等 $^{[25 \sim 28]}$. Sun等 ${ }^{[29]}$ 发现PANI改 性的氧化石墨烯(GO)具有较好的环境稳定性，对放射 性核素U(VI)、 $\mathrm{Eu}(\mathrm{III}) 、 \mathrm{Sr}(\mathrm{II})$ 和 $\mathrm{Cs}(\mathrm{I})$ 的最大吸附能力 分别高达245.1、250.8、147.2和 $184.7 \mathrm{mg} / \mathrm{g}$. 据我们所 知，目前还没有报道PANI@CNF用于放射性核素的去 除. 此外，放射性核素和PANI@CNF复合材料之间的
相互作用机制也是未知的.

因此，本文的研究目标是制备PANI@CNF复合材 料，并用扫描电子显微镜 (SEM)、透射电子显微镜 (TEM)、X射线衍射(XRD)、拉曼光谱(Raman)、傅里 叶红外光谱(FTIR)和X射线光电子能谱(XPS)分析研 究材料的物理化学性质; 通过批实验技术研究PA$\mathrm{NI} @ \mathrm{CNF}$ 复合材料在不同环境条件下对U(VI)的宏观 去除效果; 通过光谱分析研究两者之间的相互作用机 制. 该研究对于钣系元素高效去除材料的制备具有重 要的指导意义, 为铀矿开采的环境污染清理和裂变燃 料处理提供了新材料.

\section{2 实验部分}

\section{1 材料制备}

碲纳米线模板通过一种简单的水热方法制备 ${ }^{[30]}$. $1.0 \mathrm{~g}$ 聚乙烯吡咯烷酮、 $0.1844 \mathrm{~g}$ 亚碲酸纳、 $1.67 \mathrm{~mL}$ 水 合肼和 $3.33 \mathrm{~mL}$ 氨水溶解在 $33 \mathrm{~mL}$ 超纯水中, 磁性搅拌 形成混合溶液. 然后置于 $50 \mathrm{~mL}$ 的不锈钢高压反应釜 中, $180^{\circ} \mathrm{C}$ 下反应 $6 \mathrm{~h}$, 之后自然冷却到室温.

CNF通过模板导向水热法制备 ${ }^{[17]}$. 首先, 将 $42 \mathrm{~mL}$ 丙酮加入到 $8 \mathrm{~mL}$ 碲纳米线悬浮液中, 在 $6000 \mathrm{r} / \mathrm{min}$ 的条 件下离心分离得到固体. 然后, 沉淀剂被分散到 $30 \mathrm{~mL}$ 葡萄糖溶液中 ( $5 \mathrm{~g}$ 葡萄糖), 形成混合溶液. 将混合液置 于 $50 \mathrm{~mL}$ 的不锈钢高压反应釜中, $160^{\circ} \mathrm{C}$ 下反应 $16 \mathrm{~h}$, 之 后自然冷却到室温形成 $\mathrm{Te} @ \mathrm{CNF}$. 将Te@CNF材料置 于 $30 \mathrm{~mL}$ 的 $\mathrm{HCl}$ 和 $\mathrm{H}_{2} \mathrm{O}_{2}$ 混合液 (体积比为 $\mathrm{HCl}: \mathrm{H}_{2} \mathrm{O}_{2}$ : $\left.\mathrm{H}_{2} \mathrm{O}=2: 5: 23\right)$ 中, 磁力搅拌 $12 \mathrm{~h}$ 去除 $\mathrm{Te}$ 核. 用乙醇和超纯 水交替清洗材料数次, $80^{\circ} \mathrm{C}$ 下真空干燥得到 $\mathrm{CNF}$.

PANI@CNF利用原位聚合方法制备 ${ }^{[31]}$. 将1 mL苯 胺、 $20 \mathrm{~mL}$ 的 $1 \mathrm{M}$ 盐酸、 $10 \mathrm{~mL}$ 乙醇和 $30 \mathrm{~mL}$ CNF溶解 在 $50 \mathrm{~mL}$ 冰水中，搅拌 $10 \mathrm{~min}$. 然后，缓慢加入 $20 \mathrm{~mL}$ $1 \mathrm{M}$ 盐酸溶解的过硫酸铵，在冰水浴中反应 $24 \mathrm{~h}$. 之后 用乙醇和超纯水交替清洗材料数次, $60^{\circ} \mathrm{C}$ 下真空干燥 得到PANI@CNF.

\section{2 材料表征}

采用扫描电子显微镜 (SEM)、透射电子显微镜 (TEM)、X射线衍射(XRD)、拉曼光谱(Raman)、傅里 叶红外光谱(FTIR)和X射线光电子能谱(XPS)分析研 究制备材料的物理化学性质. SEM在JEM-2000VF扫 
描电子显微镜(日本)上进行．TEM在JEM-1011透射电 子显微镜上获得. 材料的相组成和相结构在 D/ $\max 2500$ 型XRD仪器上获得, 扫描范围为 $5^{\circ} \sim 70^{\circ}$. FTIR 在Nicolet Magana-IR 750型红外光谱仪(美国)上采用 溴化钾压片技术获得，波长范围为 $4000 \sim 400 \mathrm{~cm}^{-1}$. XPS光谱在ESCALAB 250 Xi XPS仪器(Thermo Fisher Scientific，美国)上获得. Raman光谱在Renishew inVia Raman型拉曼光谱仪(Renishaw, 英国) $532 \mathrm{~nm}$ 下得到.

\section{3 批吸附实验}

PANI@CNF对U(VI)的去除实验在10 mL的聚乙 烯试管中进行，系统研究 $\mathrm{pH}(2.0 \sim 11.0)$ 、背景电解质 (0.001、0.01和 $\left.0.1 \mathrm{M} \mathrm{NaNO}_{3}\right)$ 、反应时间 $(0 \sim 12 \mathrm{~h})$ 和温 度 $(298 、 313$ 和 $328 \mathrm{~K})$ 的影响. 通过加入微量的 $\mathrm{HNO}_{3}$ $(0.01 \mathrm{M})$ 或 $\mathrm{NaOH}(0.01 \mathrm{M})$ 将溶液调整到期望的 $\mathrm{pH}$. 通 过高速离心机 $10000 \mathrm{r} / \mathrm{min}$ 下离心 $10 \mathrm{~min}$ 达到固液分离 的目的. 上层清液中U(VI)的浓度采用偶氮胂III方法在 UV 2550 分光光度计上 $650 \mathrm{~nm}$ 条件下得到. U(VI)的去 除效率 $(\%)$ 和吸附能力 $\left(q_{\mathrm{e}}\right)$ 由下列方程计算得到:

$$
\begin{gathered}
\text { Sorption } \%=\frac{c_{0}-c_{\mathrm{e}}}{c_{0}} \times 100 \% \\
q_{\mathrm{e}}=\frac{\left(c_{0}-c_{e}\right) \times V}{m}
\end{gathered}
$$

\section{3 结果与讨论}

\section{1 材料表征}

采用SEM和TEM观察了制备CNF和PANI@CNF 的形态. 如图1(a)所示, 由葡萄糖生成的CNF的直径约 为 $500 \mathrm{~nm}$. CNF的表面非常光滑, 没有任何微尺度的碳 球. PANI改性之后, CNF的表面变得粗粘(图1(b, c)), 大 量的短杆状纤维在纳米材料的表面上对齐. TEM (图1 (d))图像进一步揭示出在制备好的PANI@CNF复合材 料表面含有粗粘的PANI层.

CNF和PANI@CNF的XRD如图2(a)所示. 对于原 始的 CNF, $2 \theta=21.6^{\circ}$ 处的峰被认为是石墨的(002)平面. 它的强度较弱、宽度较宽, 这主要是由于其高度无序 的性质造成的 ${ }^{[27]}$. PANI改性之后，在 $2 \theta=15^{\circ} 、 20^{\circ}$ 和 $25^{\circ}$ 处PANI@CNF上出现了 3 个典型的聚合物特征峰, 表明材料成功制备 ${ }^{[25]}$.

Raman光谱技术被用来研究制备材料的结构. 从 图2(b)中可以明显看出, CNF和PANI@CNF两个材料

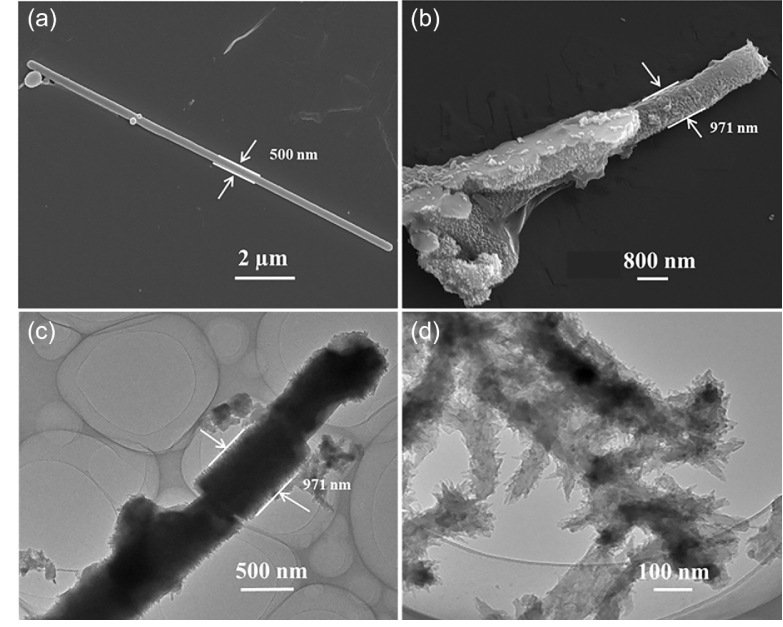

图 1 CNF (a)和PANI@CNF (b)的SEM图像; CNF (c)和 PANI@CNF (d)的TEM图像

Figure 1 SEM images of CNF (a) and PANI@CNF (b); TEM images of CNF (c) and PANI@CNF (d).
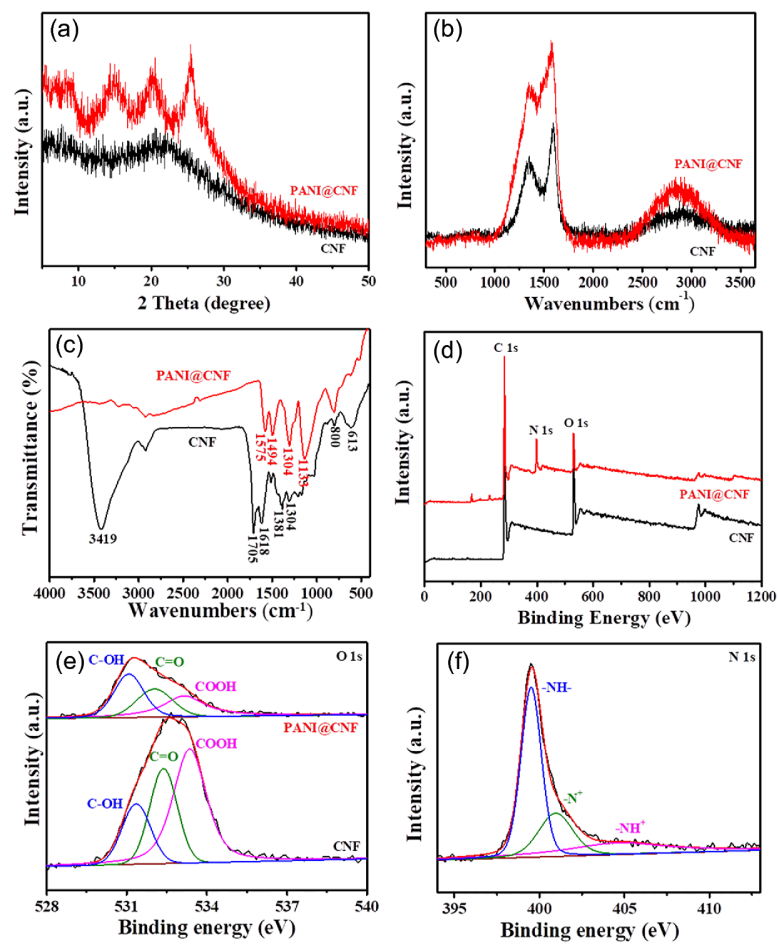

图 2 CNF和PANI@CNF的表征. (a) XRD图谱; (b) Raman光 谱; (c) FTIR光谱; (d) XPS光谱; (E) O 1s XPS光谱; (f) PANI@CNF的N 1s XPS光谱(网络版彩图)

Figure 2 Characterization of CNF and PANI@CNF samples. (a) XRD patterns; (B) Raman spectra; (C) FTIR spectra; (D) XPS spectra; (E) high resolution O 1s XPS spectra; (F) high resolution N 1s XPS spectra (color online).

均展示出明显的D峰 $\left(1350 \mathrm{~cm}^{-1}, \mathrm{sp}^{3}\right.$ 碳原子缺陷和无 序性)和 $\mathrm{G}$ 峰 $\left(\sim 1600 \mathrm{~cm}^{-1} \text {, 石墨薄片上的 } \mathrm{sp}^{2} \text { 碳原子 }\right)^{[32]}$. 
但是，与原始 $\mathrm{CNF}$ 上 $\mathrm{D}$ 峰和 $\mathrm{G}$ 峰的位置相比，PA$\mathrm{NI} @ \mathrm{CNF}$ 的D峰和G峰偏移到较低的频率, 这是由于聚 苯胺上的 $\mathrm{N}$ 原子的对电子与 $\mathrm{CNF}$ 上的碳结构发生了共 鸣, 从而影响了 $\mathrm{CNF}$ 的电子结构. $\mathrm{D}$ 峰和 $\mathrm{G}$ 峰的强度比 $\left(I_{\mathrm{D}} / I_{\mathrm{G}}\right)$ 常用于研究碳基材料的石墨化程度 ${ }^{[33]}$. CNF和 $\mathrm{PANI} @ \mathrm{CNF}$ 的 $I_{\mathrm{D}} / I_{\mathrm{G}}$ 分别为 0.70 和 0.82 . 此外, PA$\mathrm{NI} @ \mathrm{CNF}$ 在 $2800 \mathrm{~cm}^{-1}$ 出现了典型的2D峰，进一步证 明PANI改性提高了CNF材料表面的无序性.

FTIR被用于研究材料表面的官能团. 如图2(c)所 示, CNF的FTIR波谱展示出典型的 $\mathrm{C}-\mathrm{H}$ 键 $\left(613 \mathrm{~cm}^{-1}\right)$ 、 $\mathrm{C}-\mathrm{O}$ 键 $\left(1304\right.$ 和 $\left.1381 \mathrm{~cm}^{-1}\right) 、 \mathrm{sp}^{2} \quad \mathrm{C}=\mathrm{C}$ 键 $\left(1618 \mathrm{~cm}^{-1}\right)$ 、 $\mathrm{C}=\mathrm{O}$ 键 $\left(1705 \mathrm{~cm}^{-1}\right)$ 和 $\mathrm{OH}$ 伸缩振动 $\left(3419 \mathrm{~cm}^{-1}\right)^{[29]}$. 与 CNF相比，PANI@CNF的羟基和羧基明显消失，表明 聚苯胺成功复合在CNF材料表面. 此外, PANI@CNF 复合材料表现出典型的 $\mathrm{C}-\mathrm{H}$ 键 $\left(800 \mathrm{~cm}^{-1}\right)$ 、醌类化合 物的 $\mathrm{N}-\mathrm{Q}-\mathrm{N}-\mathrm{Q}$ 伸缩振动键 $\left(1133 \mathrm{~cm}^{-1}\right) 、 \mathrm{C}-\mathrm{O}$ 键 $\left(1304 \mathrm{~cm}^{-1}\right) 、 \mathrm{C}=\mathrm{C}$ 键 $\left(1494 \mathrm{~cm}^{-1}\right)$ 和 $\mathrm{N}=\mathrm{Q}=\mathrm{N}$ 键 $\left(1575 \mathrm{~cm}^{-1}\right)^{[29]}$.

XPS光谱分析进一步用于研究CNF和PANI@CNF 两个材料官能团的变化. 如图2(d)所示，两个材料都展 现出典型的C 1s $(\sim 285 \mathrm{eV})$ 和O 1s $(\sim 531 \mathrm{eV})$ 峰. 但是, 与CNF相比, PANI@CNF的O 1s峰强度明显降低, 同时 出现了新峰N 1s $(\sim 399 \mathrm{eV})$. 结果表明, 聚苯胺成功地 修饰在CNF材料表面. CNF和PANI@CNF的O 1s高分 辨光谱如图 $2(\mathrm{e})$ 所示, 可以分为 3 个含氧官能团如 $-\mathrm{COOH} \quad(\sim 533 \mathrm{eV}),-\mathrm{C}=\mathrm{O} \quad(\sim 532 \mathrm{eV})$ 和 $-\mathrm{C}-\mathrm{OH}$ $(\sim 531 \mathrm{eV})^{[31]}$. 如表1所示, $\mathrm{CNF}$ 的 $-\mathrm{COOH} 、-\mathrm{C}=\mathrm{O}$ 和 $-\mathrm{C}-\mathrm{OH}$ 分别位于 $533.4 、 532.4$ 和 $531.4 \mathrm{eV}$, 含量分别为 $51.8 \%$ 、29.1\%和 $19.1 \%$. 而PANI@CNF的 $-\mathrm{COOH}$ 、 $-\mathrm{C}=\mathrm{O}$ 和 $-\mathrm{C}-\mathrm{OH}$ 分别位于 $531.1 、 532.0$ 和 $533.2 \mathrm{eV}$ ，含
量分别为 $34.6 \%$ 、27.7\%和 $37.7 \%$. PANI $@$ CNF的N 1s 高分辨光谱如图2(f)所示，可以分为3个含氮官能团如 $-\mathrm{NH}-(399.5 \mathrm{eV}) 、-\mathrm{N}^{+}(401.0 \mathrm{eV})$ 和 $-\mathrm{NH}^{+}(404.6 \mathrm{eV})$, 含量分别为 $52.3 \% 、 22.9 \%$ 和 $24.8 \%{ }^{[31]}$.

\section{$3.2 \mathrm{pH}$ 和离子强度的影响}

通常，溶液的 $\mathrm{pH}$ 会影响 $\mathrm{U}(\mathrm{VI})$ 的物种分布以及材 料表面官能团的质子化 $\left(\right.$ 如 $\mathrm{SOH}+\mathrm{H}^{+} \rightarrow \mathrm{SOH}_{2}{ }^{+}$)过程, $\mathrm{pH}$ 和离子强度对 $\mathrm{U}(\mathrm{VI})$ 去除效果的影响如图3所示. 从图3 (a)中可以清晰地看出, U(VI)在CNF和PANI@CNF上 的去除随着 $\mathrm{pH}$ 的增加逐渐增大，在 $\mathrm{pH}>7.0$ 之后保持较 高的去除效率. U(VI)的物种分布如图3(b)所示，在 $\mathrm{pH}$ 2.0 5.0范围内, $\mathrm{U}(\mathrm{VI})$ 物种以 $\mathrm{UO}_{2}{ }^{2+} 、\left(\mathrm{UO}_{2}\right)_{2}(\mathrm{OH})_{2}{ }^{2+}$ 和 $\left(\mathrm{UO}_{2}\right)_{3}(\mathrm{OH})_{5}{ }^{+}$的形式存在. 因此, 在酸性溶液中较低的 去除率主要是由于带正电荷的U(VI)物种和正电荷的 材料表面之间的静电斥力造成的. 此外，溶液中的 $\mathrm{H}^{+}$和 $\mathrm{Na}^{+}$也会与 $\mathrm{U}(\mathrm{VI})$ 竞争材料表面的有限结合位点. 随着 $\mathrm{pH}$ 的增加, $\mathrm{U}(\mathrm{VI})$ 的去除量逐渐增大主要是由于 其与材料表面官能团形成了稳定的络合物. 在 $\mathrm{pH}>7.0$ 之后较高的去除率主要是由于 $\mathrm{U}(\mathrm{VI})$ 在材料表面形成 了柱铀矿沉淀. 此外，从图3(a)发现，同等 $\mathrm{pH}$ 条件下 $\mathrm{U}(\mathrm{VI})$ 在PANI@CNF上的去除量大于其在 $\mathrm{CNF}$ 上的去 除量, 表明PANI@CNF具有较强的络合能力.

离子强度会影响制备材料双电层的厚度和电荷密 度, 进而影响材料对放射性核素的去除效果. 一般离子 强度对外层表面络合影响较大，对内层表面络合影响 较小. 因此, 从图3(a)可以看出, U(VI)在 CNF和PA$\mathrm{NI} @ \mathrm{CNF}$ 上的去除不受硝酸钠浓度的影响. 所以, $\mathrm{U}(\mathrm{VI})$ 在CNF和PANI@CNF上的去除主要是与材料表 面官能团形成了内层表面络合物.

表 1 XPS分析不同元素的结果

Table 1 Parameters for XPS spectra of different elements

\begin{tabular}{|c|c|c|c|c|c|c|}
\hline \multirow{2}{*}{ 化学键 } & \multicolumn{3}{|c|}{$\mathrm{CNF}$} & \multicolumn{3}{|c|}{ PANI@CNF } \\
\hline & $E_{\mathrm{B}}(\mathrm{eV})$ & 峰面积 & 百分比 (\%) & $E_{\mathrm{B}}(\mathrm{eV})$ & 峰面积 & 百分比 $(\%)$ \\
\hline $\mathrm{C}-\mathrm{OH}$ & 531.4 & 1633.4 & 19.1 & 531.1 & 1310.0 & 37.7 \\
\hline $\mathrm{C}=\mathrm{O}$ & 532.4 & 2479.1 & 29.1 & 532.0 & 983.9 & 27.7 \\
\hline $\mathrm{COOH}$ & 533.4 & 4419.8 & 51.8 & 533.2 & 1231.6 & 37.7 \\
\hline$-\mathrm{NH}-$ & - & - & - & 399.5 & 1887.7 & 52.3 \\
\hline$-\mathrm{N}^{+}$ & - & - & - & 401.0 & 827.7 & 22.9 \\
\hline$-\mathrm{NH}^{+}$ & - & - & - & 404.6 & 895.7 & 24.8 \\
\hline
\end{tabular}




\section{3 反应时间的影响}

反应时间对 $\mathrm{U}(\mathrm{VI})$ 在CNF和PANI@CNF上去除效 果的影响如图4(a)所示. 随着时间的增加, U(VI)在CNF 和PANI@CNF的去除逐渐增大，分别在 2 和 0.5 h达到 平衡. 同时, U(VI)在CNF和PANI@CNF的吸附量分别 达到 75和 $125 \mathrm{mg} / \mathrm{g}$. 结果表明, PANI@CNF具有较快 的反应速率和较高的吸附量, 更加利于放射性核素的 去除.

为了进一步研究去除过程, 动力学吸附过程通过 拟一级动力学模型和拟二级动力学模型进行模拟，公 式分别如下:

$$
\begin{gathered}
\ln \left(q_{\mathrm{e}}-q_{t}\right)=\ln q_{\mathrm{e}}-k_{\mathrm{l}} t \\
\frac{t}{q_{t}}=\frac{1}{k_{2} q_{\mathrm{e}}^{2}}+\frac{1}{q_{\mathrm{e}}} t
\end{gathered}
$$

其中 $q_{t}(\mathrm{mg} / \mathrm{g})$ 和 $q_{\mathrm{e}}(\mathrm{mg} / \mathrm{g})$ 分别代表反应时间 $t(\mathrm{~h})$ 和达 到平衡时 $\mathrm{U}(\mathrm{VI})$ 在材料表面的吸附量. $k_{1}(1 / \mathrm{min})$ 和 $k_{2}$ $(\mathrm{mmol} /(\mathrm{g} \mathrm{min}))$ 分别代表拟一级动力学和拟二级动力
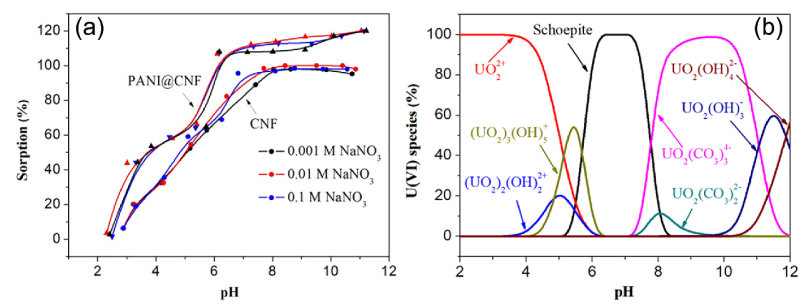

图 3 (a) $\mathrm{pH}$ 和离子强度对 $\mathrm{U}(\mathrm{VI})$ 在 $\mathrm{CNF}$ 和PANI@CNF上的 去除效果的影响. (b) U(VI)在水溶液中的物种分布. $m / V$ $=0.2 \mathrm{~g} / \mathrm{L}, T=298 \mathrm{~K}, c_{0}=30 \mathrm{mg} / \mathrm{L}$ (网络版彩图)

Figure 3 (a) Effect of $\mathrm{pH}$ value on the $\mathrm{U}(\mathrm{VI})$ adsorption to $\mathrm{CNF}$ and PANI@CNF at various $\mathrm{NaNO}_{3}$ concentrations at $m / V=0.2 \mathrm{~g} / \mathrm{L} ; T=$ $298 \mathrm{~K} ; c_{0}=30 \mathrm{mg} / \mathrm{L}$. (b) The distribution of various species of $\mathrm{U}(\mathrm{VI})$ as a function of $\mathrm{pH}$ (color online).
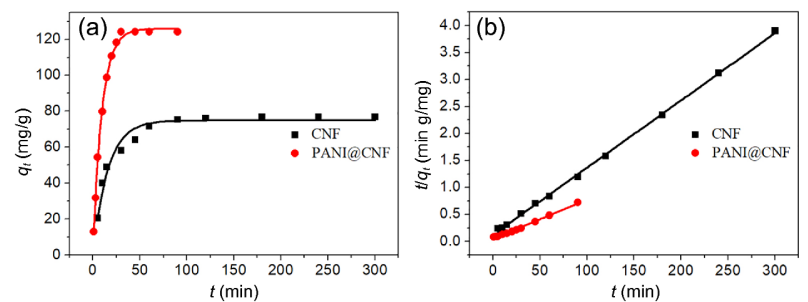

图 4 (a) 反应时间对 $\mathrm{U}(\mathrm{VI})$ 在 $\mathrm{CNF}$ 和PANI@CNF上去除效 果的影响. (b) 拟二级动力学模型拟合曲线. $m / V=0.2 \mathrm{~g} / \mathrm{L}, T$ $=298 \mathrm{~K}, I=0.01 \mathrm{M} \mathrm{NaNO}_{3}, \mathrm{pH}=5.0 \pm 0.1$ (网络版彩图)

Figure 4 (a) Adsorption kinetics of U(VI) on CNF and PANI@CNF. (b) Pseudo-second order kinetic plots of U(VI). $m / V=0.2 \mathrm{~g} / \mathrm{L} ; T=298 \mathrm{~K}$; $I=0.01 \mathrm{M} \mathrm{NaNO}_{3} ; \mathrm{pH}=5.0 \pm 0.1$ (color online).
学反应常数. 从图 $4(b)$ 和表 2 中可以看出, $R_{1}{ }^{2}<0.995$ 和 $R_{2}^{2}>0.997$ 说明 $\mathrm{U}(\mathrm{VI})$ 的去除过程符合拟二级动力学模 型, 表明U(VI)在去除过程主要受化学作用控制而不是 物理反应过程 ${ }^{[34]}$.

\section{4 反应温度的影响}

在298、313和328 K温度下, U(VI)在CNF和PA$\mathrm{NI} @ \mathrm{CNF}$ 上的吸附等温线如图5所示. 随着温度的升 高, U(VI)的吸附量明显增加, 表明高温利于 $\mathrm{U}(\mathrm{VI})$ 的 去除, 吸附过程是吸热反应. 为了进一步研究反应本 质, 吸附等温线通过Langmuir ${ }^{[35]}$ 和Freundlich ${ }^{[36]}$ 等温式 进行拟合, 公式如下:

$$
\begin{aligned}
& \frac{c_{\mathrm{e}}}{q_{\mathrm{e}}}=\frac{1}{q_{\max } k_{\mathrm{L}}}+\frac{c_{\mathrm{e}}}{q_{\max }} \\
& \ln q_{\mathrm{e}}=\ln K_{\mathrm{F}}+\frac{1}{n} \ln c_{\mathrm{e}}
\end{aligned}
$$

其中, $q_{\mathrm{e}}\left(\mathrm{mg} / \mathrm{g}\right.$ )和 $q_{\text {max }}(\mathrm{mg} / \mathrm{g})$ 分别代表 $\mathrm{U}(\mathrm{VI})$ 在吸附剂 上的吸附量和最大吸附量, $c_{\mathrm{e}}(\mathrm{mg} / \mathrm{L})$ 代表 $\mathrm{U}(\mathrm{VI})$ 的平衡 浓度, $K_{\mathrm{L}}$ 代表Langmuir常数, $n$ 代表吸附强度, $K_{F}$ 代表 Freundlich常数. 各参数拟合结果如表3. 结果表明, Langmuir模型更加符合去除过程, 说明U(VI)的去除是 单分子层均匀覆盖过程, 去除机理是化学作用. $298 \mathrm{~K}$ 条件下U(VI)在PANI@CNF上的去除量 $(319.4 \mathrm{mg} / \mathrm{g})$ 高 于其在 $\mathrm{CNF}$ 上的去除量 $(133.9 \mathrm{mg} / \mathrm{g})$. 此外, PA$\mathrm{NI} @ \mathrm{CNF}$ 材料与其他材料的性质对比见表 $4^{[37 \sim 45]}$. 可 以看出, PANI@CNF材料具有较高的吸附量, 这主要 是由于其表面含有丰富的含氧官能团和含氮官能团, 能够与 $\mathrm{U}(\mathrm{VI})$ 形成稳定的络合物.

$\mathrm{U}(\mathrm{VI})$ 在PANI@CNF上吸附的热力学参数, 如吉布 斯自由能变化 $(\Delta G, \mathrm{~kJ} / \mathrm{mol}) 、$ 标准焓变 $(\Delta H, \mathrm{~kJ} / \mathrm{mol})$ 和
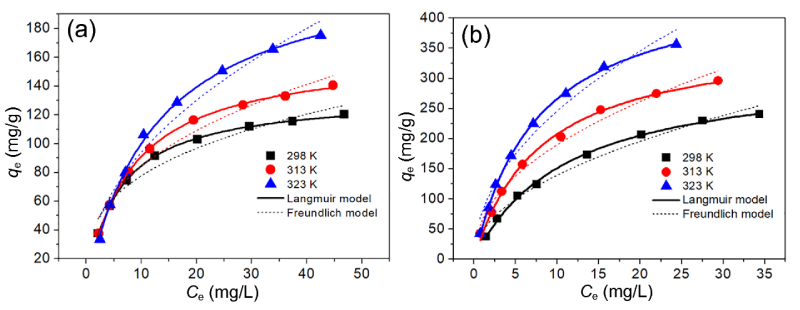

图 $5 \mathrm{U}(\mathrm{VI})$ 在CNF (a) 和PANI@CNF (b) 上的吸附等温线. $m /$ $V=0.2 \mathrm{~g} / \mathrm{L}, \mathrm{pH}=5.0 \pm 0.1$ (网络版彩图)

Figure 5 Adsorption isotherms of U(VI) on CNF (a) and PANI@CNF (b) under three temperatures. $m / V=0.2 \mathrm{~g} / \mathrm{L} ; \mathrm{pH}=5.0 \pm 0.1$ (color online). 
表 $2 \mathrm{U}(\mathrm{VI})$ 在CNF和PANI@CNF上的吸附动力学参数

Table 2 Parameters of kinetic models for U(VI) adsorption on CNF and PANI@CNF

\begin{tabular}{|c|c|c|c|c|c|c|}
\hline \multirow{2}{*}{ 吸附剂 } & \multicolumn{3}{|c|}{ 拟一级动力学模型 } & \multicolumn{3}{|c|}{ 拟二级动力学模型 } \\
\hline & $k_{1}(1 / \mathrm{min})$ & $q_{\mathrm{e}}(\mathrm{mg} / \mathrm{g})$ & $R^{2}$ & $k_{2}(\mathrm{~g} /(\operatorname{mg} \min ))$ & $q_{\mathrm{e}}(\mathrm{mg} / \mathrm{g})$ & $R^{2}$ \\
\hline $\mathrm{CNF}$ & 0.064 & 74.9 & 0.9611 & 0.001 & 80.6 & 0.9992 \\
\hline PANI@CNF & 0.105 & 125.9 & 0.9918 & 0.001 & 138.9 & 0.9973 \\
\hline
\end{tabular}

表 $3 \mathrm{U}(\mathrm{VI})$ 在CNF和PANI@CNF上吸附的Langmuir和Freundlich模型参数

Table 3 Parameters of Langmuir and Freundlich isotherm models for U(VI) adsorption on CNF and PANI@CNF

\begin{tabular}{|c|c|c|c|c|c|c|c|}
\hline \multirow{2}{*}{ 吸附剂 } & \multirow{2}{*}{$T(\mathrm{~K})$} & \multicolumn{3}{|c|}{ Langmuir 模型 } & \multicolumn{3}{|c|}{ Freundlich 模型 } \\
\hline & & $q_{\max }(\mathrm{mg} / \mathrm{g})$ & $K_{\mathrm{L}}(\mathrm{L} / \mathrm{mg})$ & $R^{2}$ & $K_{\mathrm{F}}(\mathrm{mg} / \mathrm{g}) /(\mathrm{mg} / \mathrm{L})^{n}$ & $n$ & $R^{2}$ \\
\hline \multirow{3}{*}{$\mathrm{CNF}$} & 298 & 133.9 & 0.17 & 0.999 & 37.46 & 0.32 & 0.949 \\
\hline & 313 & 163.5 & 0.13 & 0.999 & 35.82 & 0.37 & 0.960 \\
\hline & 323 & 228.9 & 0.08 & 0.998 & 31.98 & 0.47 & 0.962 \\
\hline \multirow{3}{*}{ PANI@CNF } & 298 & 319.4 & 0.09 & 0.998 & 45.11 & 0.49 & 0.977 \\
\hline & 313 & 373.7 & 0.13 & 0.995 & 65.26 & 0.46 & 0.979 \\
\hline & 323 & 469.5 & 0.13 & 0.999 & 78.77 & 0.49 & 0.969 \\
\hline
\end{tabular}

表 $4 \mathrm{U}(\mathrm{VI})$ 在不同吸附剂上吸附能力的对比

Table 4 Comparison of uptake capacity of different materials

\begin{tabular}{|c|c|c|c|c|c|}
\hline \multirow{2}{*}{ 吸附剂 } & \multicolumn{3}{|c|}{ 实验条件 } & \multirow{2}{*}{$q_{\max }(\mathrm{mg} / \mathrm{g})$} & \multirow{2}{*}{ Ref } \\
\hline & $\mathrm{pH}$ & $I$ & $t(\min )$ & & \\
\hline Ca-Mg-Al-LDH & 5.0 & $0.01 \mathrm{M} \mathrm{NaNO}_{3}$ & 1200 & 132.5 & {$[37]$} \\
\hline $\mathrm{Fe}_{3} \mathrm{O}_{4} @ \mathrm{TNS}$ & 5.0 & $0.01 \mathrm{M} \mathrm{NaNO}_{3}$ & 600 & 68.6 & {$[38]$} \\
\hline $\mathrm{Fe}_{3} \mathrm{O}_{4} / \mathrm{GO}$ 复合材料 & 5.5 & $0.1 \mathrm{M} \mathrm{Na}_{2} \mathrm{CO}_{3}$ & 240 & 69.5 & [39] \\
\hline N-doped $\mathrm{Fe} / \mathrm{Fe}_{3} \mathrm{C} @ \mathrm{C}$ & 6.0 & $0.1 \mathrm{M} \mathrm{Na}_{2} \mathrm{CO}_{3}$ & 120 & 203.0 & {$[40]$} \\
\hline $\mathrm{MnO}_{2} @ \mathrm{PPy}$ & 5.0 & $0.01 \mathrm{M} \mathrm{NaCl}$ & 300 & 63.0 & {$[41]$} \\
\hline $1-\mathrm{C}_{3} \mathrm{~N}_{4} / \mathrm{PDA} / \mathrm{PEI}_{3}$ & 5.0 & $0.01 \mathrm{M} \mathrm{NaClO}_{4}$ & 1200 & 61.7 & {$[42]$} \\
\hline MMT@C & 4.9 & $0.01 \mathrm{M} \mathrm{NaClO}_{4}$ & 300 & 66.2 & {$[43]$} \\
\hline $\mathrm{MnFe}_{2} \mathrm{O}_{4}$ & 5.0 & $0.1 \mathrm{M} \mathrm{NaNO}_{3}$ & 240 & 119.9 & [44] \\
\hline CS@LDH & 5.0 & $0.01 \mathrm{M} \mathrm{NaNO}_{3}$ & 180 & 142.8 & {$[45]$} \\
\hline $\mathrm{CNF}$ & 5.0 & $0.01 \mathrm{M} \mathrm{NaNO}_{3}$ & 120 & 133.9 & 本文 \\
\hline PANI@CNF & 5.0 & $0.01 \mathrm{M} \mathrm{NaNO}_{3}$ & 30 & 319.4 & Th \\
\hline
\end{tabular}

标准熵变 $(\Delta S, \mathrm{~J} /(\mathrm{K} \mathrm{mol}))$ 通过吸附等温线得到. $\Delta G$ 通过 以下公式得到:

$$
\begin{gathered}
\Delta G=-R T \ln K_{C} \\
K_{C}=10^{6} \times K_{\mathrm{L}}
\end{gathered}
$$

其中 $K_{C}$ 代表吸附平衡常数, $K_{\mathrm{L}}$ 代表吸附剂和吸附质之
间的亲和力常数.

$\Delta S$ 通过以下公式得到:

$$
\ln K_{C}=\frac{-\Delta H}{R} \times \frac{1}{T}+\frac{\Delta S}{R}
$$

其中 $R$ 是理想气体常数 $(8.314 \mathrm{~J} /(\mathrm{mol} \mathrm{K})), T(\mathrm{~K})$ 是开尔 文温度. 拟合得到的热力学参数见表 $5 . \Delta G$ 是负的, 表 
表 $5 \mathrm{U}(\mathrm{VI})$ 在CNF和PANI@CNF上吸附的热力学参数

Table 5 Thermodynamic parameters for the adsorption of U(VI) on CNF and PANI@CNF

\begin{tabular}{ccccc}
\hline 吸附剂 & $T(\mathrm{~K})$ & $\Delta G(\mathrm{KJ} / \mathrm{mol})$ & $\Delta H(\mathrm{KJ} / \mathrm{mol})$ & $\Delta S(\mathrm{~J} /(\mathrm{mol} \mathrm{K}))$ \\
\hline & 298 & -29.8 & & 32.4 \\
CNF & 313 & -30.6 & & 128.9 \\
& 328 & -30.8 & 10.1 & 128.9 \\
\hline
\end{tabular}

明吸附过程是吸热反应 ${ }^{[46]}$. 且随着温度的升高, $\Delta G$ 越 来越负，表明高温利于放射性核素的去除. $\Delta H$ 是正的， 表明吸附过程是自发进行的. $\Delta S$ 是正的, 表明 $\mathrm{U}(\mathrm{VI})$ 的 吸附过程增加了固液界面的无序性.

\section{4 结论}

本研究成功制备了PANI改性的CNF材料, 并将其 用于放射性核素U(VI)的高效去除.PANI@CNF对
$\mathrm{U}(\mathrm{VI})$ 的去除受 $\mathrm{pH}$ 的影响较大, 而离子强度没有影响, 表明作用机理主要是内层表面络合. PANI@CNF对 $\mathrm{U}(\mathrm{VI})$ 的吸附符合拟二级动力学模型, 且反应在 $0.5 \mathrm{~h}$ 内 快速达到平衡. U(VI)在PANI@CNF上的吸附符合 Langmuir等温线, 其最大吸附量为 $319.4 \mathrm{mg} / \mathrm{g}$. FTIR和 XPS 分析发现U(VI)在PANI@CNF上的高效去除主要 是由于其表面丰富的含氧和含氮官能团. PANI@CNF 吸附剂有望作为潜在材料用于放射性核素的处理和 处置.

\section{参考文献}

1 Pang H, Wang X, Yao W, Yu S, Wang X. Sci Sin-Chim, 2018, 48: 58-73 (in Chinese) [庞宏伟, 王祥学, 姚文, 于淑君, 王祥科. 中国科学: 化学, 2018, 48: 58-73]

2 Ai YJ, Liu Y, Lan WY, Jin JR, Xing JL, Zou YD, Zhao CF, Wang XK. Chem Eng J, 2018, 343: 460-466

3 Li J, Wang X, Zhao G, Chen C, Chai Z, Alsaedi A, Hayat T, Wang X. Chem Soc Rev, 2018, 47: 2322-2356

4 Yang S, Wang X, Chen Z, Li, Wei B, Wang X. Prog Chem, 2018, 30: 225-242 (in Chinese) [杨姗也, 王祥学, 陈中山, 李倩, 韦埥堑, 王祥科. 化 学进展, 2018, 30: 225-242]

5 Chen H, Huang S, Zhang Z, Liu Y, Wang X. Acta Chim Sin, 2017, 75: 560 (in Chinese) [陈海军, 黄舒怡, 张志宾, 刘云海, 王祥科. 化学学报, 2017, 75: 560-574]

6 Yu S, Wang J, Song S, Sun K, Li J, Wang X, Chen Z, Wang X. Sci China Chem, 2017, 60: 415-422

7 Yu S, Wang X, Yang S, Sheng G, Alsaedi A, Hayat T, Wang X. Sci China Chem, 2016, 60: 170-187

8 Yu S, Wang X, Pang H, Zhang R, Song W, Fu D, Hayat T, Wang X. Chem Eng J, 2018, 333: 343-360

9 Gu P, Zhang S, Li X, Wang X, Wen T, Jehan R, Alsaedi A, Hayat T, Wang X. Environ Pollut, 2018, 240: 493-505

10 Wang X, Liu Y, Pang H, Yu S, Ai Y, Ma X, Song G, Hayat T, Alsaedi A, Wang X. Chem Eng J, 2018, 344: 380-390

11 Wang X, Chen Z, Wang X. Sci China Chem, 2015, 58: 1766-1773

12 Li X, Liu Y, Zhang C, Wen T, Zhuang L, Wang X, Song G, Chen D, Ai Y, Hayat T, Wang X. Chem Eng J, 2018, 336: 241-252

13 Pang H, Huang S, Wu Y, Yang D, Wang X, Yu S, Chen Z, Alsaedi A, Hayat T, Wang X. Inorg Chem Front, 2018,50

14 Tan X, Fang M, Tan L, Liu H, Ye X, Hayat T, Wang X. Environ Sci-Nano, 2018, 5: 1140-1149

15 Wang P, Yin L, Wang X, Zhao G, Yu S, Song G, Xie J, Alsaedi A, Hayat T, Wang X. J Environ Manage, 2018, 217: 468-477

16 Liang HW, Wu ZY, Chen LF, Li C, Yu SH. Nano Energy, 2015, 11: 366-376

17 Liang HW, Guan QF, Chen LF, Zhu Z, Zhang WJ, Yu SH. Angew Chem Int Ed, 2012, 51: 5101-5105

18 Bolisetty S, Mezzenga R. Nat Nanotech, 2016, 11: 365-371 
Patiño Y, Díaz E, Ordóñez S. Chemosphere, 2015, 119: S124-S130

Yu S, Liu Y, Ai Y, Wang X, Zhang R, Chen Z, Chen Z, Zhao G, Wang X. Environ Pollut, 2018, 242: 1-11

Sun Y, Wu ZY, Wang X, Ding C, Cheng W, Yu SH, Wang X. Environ Sci Technol, 2016, 50: 4459-4467

Sun Y, Wang X, Ding C, Cheng W, Chen C, Hayat T, Alsaedi A, Hu J, Wang X. ACS Sustain Chem Eng, 2016, 4: 4608-4616

Sun Y, Lu S, Wang X, Xu C, Li J, Chen C, Chen J, Hayat T, Alsaedi A, Alharbi NS, Wang X. Environ Sci Technol, 2017, 51: 12274-12282

Wang J, Deng B, Chen H, Wang X, Zheng J. Environ Sci Technol, 2009, 43: 5223-5228

Zhu K, Gao Y, Tan X, Chen C. ACS Sustain Chem Eng, 2016, 4: 4361-4369

Zhao D, Chen L, Xu M, Feng S, Ding Y, Wakeel M, Alharbi NS, Chen C. ACS Sustain Chem Eng, 2017, 5: 10290-10297

Zhu K, Chen C, Xu M, Chen K, Tan X, Wakeel M, Alharbi NS. Chem Eng J, 2018, 331: 395-405

Zhang J, Han J, Wang M, Guo R. J Mater Chem A, 2017, 5: 4058-4066

Sun Y, Shao D, Chen C, Yang S, Wang X. Environ Sci Technol, 2013, 47: 9904-9910

Qian HS, Yu SH, Luo LB, Gong JY, Fei LF, Liu XM. Chem Mater, 2006, 18: 2102-2108

Shao D, Hou G, Li J, Wen T, Ren X, Wang X. Chem Eng J, 2014, 255: 604-612

Yu S, Wang X, Yao W, Wang J, Ji Y, Ai Y, Alsaedi A, Hayat T, Wang X. Environ Sci Technol, 2017, 51: 3278-3286

Yu S, Wang X, Ai Y, Tan X, Hayat T, Hu W, Wang X. J Mater Chem A, 2016, 4: 5654-5662

Yao W, Yu S, Wang J, Zou Y, Lu S, Ai Y, Alharbi NS, Alsaedi A, Hayat T, Wang X. Chem Eng J, 2017, 307: 476-486

Tran HN, You SJ, Hosseini-Bandegharaei A, Chao HP. Water Res, 2017, 120: 88-116

Wu Y, Pang H, Yao W, Wang X, Yu S, Yu Z, Wang X. Sci Bull, 2018, doi: 10.1016/Jscib.2018.05.021

Zou Y, Wang X, Wu F, Yu S, Hu Y, Song W, Liu Y, Wang H, Hayat T, Wang X. ACS Sustain Chem Eng, 2016, 5: 1173-1185

Yin L, Song S, Wang X, Niu F, Ma R, Yu S, Wen T, Chen Y, Hayat T, Alsaedi A, Wang X. Environ Pollut, 2018, 238: 725-738

Zong P, Wang S, Zhao Y, Wang H, Pan H, He C. Chem Eng J, 2013, 220: 45-52

Wen T, Wang X, Wang J, Chen Z, Li J, Hu J, Hayat T, Alsaedi A, Grambow B, Wang X. Inorg Chem Front, 2016, 3: 1227-1235

Yao W, Wu Y, Pang H, Wang X, Yu S, Wang X. Sci China Chem, 2018, 61: 812-823

Wang P, Yin L, Wang J, Xu C, Liang Y, Yao W, Wang X, Yu S, Chen J, Sun Y, Wang X. Chem Eng J, 2017, 326: 863-874

Zhang R, Chen C, Li J, Wang X. Appl Surf Sci, 2015, 349: 129-137

Hu Y, Zhao C, Yin L, Wen T, Yang Y, Ai Y, Wang X. Chem Eng J, 2018, 349: 347-357

Wang X, Yu S, Wu Y, Pang H, Yu S, Chen Z, Hou J, Alsaedi A, Hayat T, Wang S. Chem Eng J, 2018, 342: 321-330

Yu S, Yin L, Pang H, Wu Y, Wang X, Zhang P, Hu B, Chen Z, Wang X. Chem Eng J, 2018, doi: 10.1016/JceJ2018.07.033 


\title{
Highly efficient removal of radioactive uranium on polyaniline modified carbon nanofiber composites
}

\author{
Sufei Wang ${ }^{1}$, Shujun $\mathrm{Yu}^{2}$, Yihan $\mathrm{Wu}^{2}$, Hongwei Pang ${ }^{2}$, Zhongshan Chen ${ }^{2}$, Xiangxue Wang ${ }^{3 *}$ \\ ${ }^{1}$ The Experimental High School Attached To Beijing Normal University, Beijing 100032, China \\ ${ }^{2}$ College of Environmental Science and Engineering, North China Electric Power University, Beijing 102206, China \\ ${ }^{3}$ Department of Environmental Science and Engineering, North China Electric Power University, Baoding 071003, China \\ *Corresponding author (email: xxwang@ncepu.edu.cn)
}

\begin{abstract}
In this article, polyaniline modified carbon nanofiber (PANI@CNF) composites were synthesized by in situ polymerization technique, and applied to highly efficient removal of radioactive uranium (U(VI)) from aqueous solutions. The prepared materials had abundant functional groups and excellent physical and chemical properties, which was confirmed by scanning electron microscope (SEM), transmission electron microscope (TEM), X-ray diffraction (XRD), Fourier transform infrared spectroscopy (FTIR) and X-ray photoelectron spectroscopy (XPS). The adsorption of $\mathrm{U}(\mathrm{VI})$ from aqueous solutions as a function of $\mathrm{pH}$, ionic strength, reaction time and temperature on the materials were investigated by batch experiments. The elimination of $\mathrm{U}(\mathrm{VI})$ was strongly dependent on $\mathrm{pH}$ but independent of ionic strength, indicating that the interaction was mainly dominant by inner-sphere surface complexation. The adsorption kinetics were well simulated by pseudo-second-order model and reached reaction equilibrium within $30 \mathrm{~min}$. The adsorption isotherms were well described by Langmuir isotherm model, implying that the elimination of U(VI) was monolayer coverage. At $\mathrm{pH}=5.0$ and $T=298 \mathrm{~K}$, the maximum adsorption capacity of U(VI) on PANI@CNF (319.4 mg/g) was higher than those of U(VI) on CNF $(133.9 \mathrm{mg} / \mathrm{g})$, which was attributed to the synergistic effects between oxygen functional groups and nitrogen functional groups. Such a facile preparation method and efficient removal performance highlighted the application of PANI@CNF as a candidate for rapid and efficient radionuclide contamination's elimination in practical applications.
\end{abstract}

Keywords: carbon nanomaterial, polyaniline, uranium, spectrum analysis, adsorption

doi: $10.1360 / \mathrm{N} 032018-00125$ 